\section{Management of gastro- oesophageal reflux and regurgitation during anaesthesia in a dog, using a supraglottic airway device (SGAD) with an integrated oesophageal drain}

\section{Ivan Crotaz}

Kynoch Vets, Bracknell, United Kingdom

\section{OBJECTIVES}

Gastro-oesophageal reflux and regurgitation are common and serious anaesthetic complications in dogs. Supraglottic airway devices (SGADs) form a seal over the larynx. The device tip enters the oesophagus and incorporates an oesophageal drainage tube to channel regurgitate away from the airway.

This case study describes the first use of a species specific dog SGAD with an anaesthetic case in which a high volume regurgitation event occurred.

\section{METHODS}

A $26 \mathrm{~kg}$ male Staffordshire Bull Terrier was undergoing general anaesthesia for a neutering procedure. Informed consent had been obtained from a representative of the rescue centre responsible for this dog. Following routine premedication and induction, a size 4 dog SGAD was placed into the pharynx. The device was connected to a Humphreys ADE circuit to supply oxygen and isoflurane. Multiparameter monitoring was used during the procedure.

The dog was positioned in dorsal recumbency on a surgical bean bag.

\section{RESULTS}

As surgery commenced, a noise was audible from the neck region. Approximately $100 \mathrm{ml}$ of brown fluid exited the gastric channel. A gastric drain tube was inserted into the gastric channel of the $v$-gel, allowing removal of material and oesophageal flushing with sterile saline to remove remaining debris. Intra-airway endoscopy demonstrated that no regurgitate had entered the airway channel.

\section{STATEMENT (CONCLUSIONS)}

This demonstrates that regurgitation can occur without warning during anaesthesia in the dog and that placing a drainage channel in the oesophagus can help to channel unexpected material away from the airway. An integrated drainage channel can also make placement of an oesophageal or gastric drainage tube easier.

\section{A case of recurrent obstructive fibrinous tracheal pseudomembrane in a dog}

\section{Louise Dawson'1, Kirsty Baird²}

1 IDEXX Laboratories UK, Wetherby, United Kingdom

2 Hird \& Partners LLP, Halifax, United Kingdom

\section{OBJECTIVES}

Recurrent obstructive fibrinous tracheal pseudomembranes are a rare complication of endotracheal intubation in humans. Only one case is reported in the veterinary literature to our knowledge. The clinical presentation and histopathological findings in another case is described in this case report.

\section{METHODS}

Samples from the trachea of a 9 year-old border terrier were submitted to IDEXX Laboratories, Inc. for histopathological evaluation. The patient presented with respiratory difficulties following a previous general anaesthetic.

\section{RESULTS}

Histopathology was consistent with aggregates of debris, fibrin and inflammatory cells, consistent with fibrinous tracheal pseudomembrane. The dog recovered uneventfully following removal of the intratracheal fibrinous material.

\section{STATEMENT (CONCLUSIONS)}

Recurrent obstructive fibrinous tracheal pseudomembranes are a rare complication of endotracheal intubation and should be on the differential diagnosis list for dogs presenting with dyspnoea following a recent general anaesthetic. 